\title{
Functional characterization of Fusarium verticillioides CPP1, a gene encoding a putative protein phosphatase $2 \mathrm{~A}$ catalytic subunit
}

\author{
Yoon-E Choi and Won-Bo Shim
}

Correspondence

Won-Bo Shim

wbshim@tamu.edu

Received 9 July 2007

Revised 11 September 2007

Accepted 13 September 2007

\author{
Department of Plant Pathology and Microbiology, Program for the Biology of Filamentous Fungi, \\ Texas A\&M University, College Station, TX 77843-2132, USA
}

\begin{abstract}
Fusarium verticillioides produces the mycotoxin fumonisin $\mathrm{B}_{1}\left(\mathrm{FB}_{1}\right)$ on maize kernels. In this study, we identified a putative protein phosphatase gene CPP1 in F. verticillioides, and investigated its role in $\mathrm{FB}_{1}$ regulation. Previous work has shown that CPP1 expression is elevated in an $\mathrm{FB}_{1}$-suppressing genetic background. Thus, we hypothesized that $\mathrm{CPP} 1$ is negatively associated with $\mathrm{FB}_{1}$ production. To test this hypothesis, we generated a CPP1 knockout mutant, PP179, and studied the effects of gene deletion on $\mathrm{FB}_{1}$ biosynthesis and fungal development. PP179 showed elevated expression of FUM genes, and in turn produced higher levels of $\mathrm{FB}_{1}$ than the wild-type progenitor. Other significant mutant phenotypes included reduced radial growth on agar plates, reduced conidia germination rates, significantly increased macroconidia formation, and hyphal swelling. To verify that these phenotypes were directly due to CPP1 deletion, we complemented PP179 with the wild-type CPP1 gene. The complemented strain PPC4 showed FUM1 expression and $\mathrm{FB}_{1}$ production similar to that of the wild-type, providing evidence that CPP1 is negatively associated with $\mathrm{FB}_{1}$ biosynthesis. Other PP179 phenotypes, such as macroconidiation and hyphal swelling, were also restored to that of wild-type progenitor. Furthermore, we complemented F. verticillioides PP179 strain with Neurospora crassa wild-type ppe-1 gene, demonstrating that Cpp1 and PPE-1 proteins are functionally conserved. Pleiotropic effects of CPP1 deletion led us to hypothesize that CPP1 is associated with multiple downstream signalling pathways in $F$. verticillioides. Identification and functional characterization of downstream Cpp1-interacting proteins are necessary to better understand the complex regulatory mechanisms associated with Cpp1.
\end{abstract}

\section{INTRODUCTION}

The fungal pathogen Fusarium verticillioides (Sacc.) Nirenburg (teleomorph Gibberella moniliformis Wineland) has been the topic of extensive research based on its production of the mycotoxin fumonisin $\mathrm{B}_{1}\left(\mathrm{FB}_{1}\right)$ on maize. $\mathrm{FB}_{1}$ is a potent carcinogen, and ingestion of fumonisin-contaminated corn has been linked to a variety of illnesses, including leukoencephalomalasia and neural tube defects in animals (Gelderblom et al., 1988; Marasas, 2001; Minorsky, 2002; Missmer et al., 2006). Due to these

Abbreviations: $\mathrm{FB}_{1}$ fumonisin $\mathrm{B}_{1}$, ; PKS, polyketide synthase; qRT-PCR, quantitative real-time RT-PCR; SSH, subtractive suppression hybridization, WT, wild-type.

The GenBank/EMBL/DDBJ accession number for the nucleotide sequence of CPP1 is D0924537.

A description of PP179 complementation using the YEC3 construct (section 1) and a description of how exogenous application of protein phosphatase inhibitor okadaic acid did not induce hyphal swelling in Fusarium verticillioides (section 2) are available as supplementary data with the online version of this paper. serious concerns, the US Food and Drug Administration has established guidelines for the fumonisin level in feed and foodstuff (Park \& Troxell, 2002). Significant progress has been made in elucidating the fumonisin biosynthetic pathway, and regulatory mechanisms associated with the toxin production. The biosynthetic gene cluster, including the polyketide synthase (PKS) gene FUM1 (Proctor et al., 1999, 2003; Seo et al., 2001), and a number of regulatory genes involved in fumonisin biosynthesis, namely $P A C 1$, FCC1 and ZFR1, have been identified and characterized (Flaherty et al., 2003; Flaherty \& Woloshuk, 2004; Shim \& Woloshuk, 2001). However, these genes do not show clear epistatic relationships, and therefore it is conceivable that multiple signalling pathways are associated with fumonisin regulation. Moreover, a putative regulatory gene, FUM21, within the FUM cluster, has recently been identified and characterized, but it was shown that the deletion of the FUM21 gene did not completely block fumonisin biosynthesis (Brown et al., 2007). That report suggested that transcriptional regulation of FUM genes can also be affected by putative regulatory gene(s) outside the FUM 
cluster. Additionally, the complexity of fumonisin regulation is further enhanced by a variety of physiological and nutritional conditions, notably acidic $\mathrm{pH}$ and nitrogen stress, which are known to favour or perhaps trigger fumonisin biosynthesis (Flaherty et al., 2003; Shim \& Woloshuk, 1999).

In an effort to identify additional genes associated with fumonisin regulation, Shim \& Woloshuk (2001) constructed a subtractive suppression hybridization ( $\mathrm{SSH}$ ) cDNA library to screen genes that are differentially expressed during fumonisin biosynthesis in F. verticillioides wild-type (WT) and FT536 strains. FT536 harbours a mutation in the $F C C 1$ gene, which encodes a type-C cyclin. Mutation of FCC1 results in reduced conidiation and drastic reduction in fumonisin production when grown on maize kernels (Shim \& Woloshuk, 2001). Microarray analysis using $F$. verticillioides oligoarrays has further verified genes that are expressed concomitantly with fumonisin production (Pirttilä et al., 2004). A number of these genes have been selected for characterization of their role in fumonisin biosynthesis. For instance, cDNA encoding a putative zinc binuclear cluster-type transcription factor, later designated ZFR1, has been shown to be positively associated with fumonisin biosynthesis (Flaherty \& Woloshuk, 2004). Deletion of ZFR1 in F. verticillioides has been shown to result in greater than $90 \%$ reduction in fumonisin production when compared with the WT. Also, Sagaram et al. (2006) have recently demonstrated that GBP1, a gene originally identified in the FT536 SSH cDNA library, encodes a monomeric G-protein that is negatively associated with fumonisin biosynthesis.

In the FT536 SSH cDNA library, we isolated a $300 \mathrm{bp}$ cDNA fragment (ft536_0_M14) that shows a high level of similarity to a Neurospora crassa gene that encodes a probable cell-shape-control protein phosphatase 2A catalytic subunit, PPE-1 (E value, 5e-45). Protein phosphatases catalyse the dephosphorylation of specific substrates that are important for processing various biological and cellular functions (Ceulemans \& Bollen, 2004; Dickman \& Yarden, 1999; Hunter, 1995), and this catalytic action is known to de-activate signalling pathways induced by a variety of protein kinases (Hunter, 1995). In Arabidopsis, for instance, protein phosphatase type $2 \mathrm{C}$ is involved in the negative regulation of the abscisic acid signalling pathway (Saez et al., 2004). Overall, we have limited understanding of the role of protein phosphatases in filamentous fungi, particularly in association with secondary metabolism. Moreover, protein phosphatases in F. verticillioides have not been functionally characterized to date.

Since cDNA ft536_ 0_M14 was identified among a collection of genes upregulated during fumonisin suppression, we hypothesize that this gene, designated CPP1 (probable cell-shape-control protein phosphatase), is negatively associated with fumonisin biosynthesis in $F$. verticillioides. To test this hypothesis, we generated a $C P P 1$ gene deletion mutant, and investigated the role of $C P P 1$ in
F. verticillioides. In this study, we report that deletion of $C P P 1$ results in elevated fumonisin production via derepression (or upregulation) of FUM1 expression. We also show that mutation in CPP1 results in pleiotropic phenotypes, suggesting that $C P P 1$ is associated with multiple signalling pathways that control fungal development and differentiation. Lastly, we demonstrate that $N$. crassa WT ppe-1 gene can complement $F$. verticillioides $C P P 1$ gene deletion.

\section{METHODS}

Fungal strain, and culture media. F. verticillioides strain 7600 (Fungal Genetics Stock Center, University of Missouri, Kansas City, MO, USA) was stored in $20 \%(\mathrm{v} / \mathrm{v})$ glycerin at $-80{ }^{\circ} \mathrm{C}$. Conidia were produced for inoculum by growing the fungus on V8 juice agar (200 ml V8 juice $1^{-1}, 3 \mathrm{~g} \mathrm{CaCO}_{3} \mathrm{l}^{-1}$, and $20 \mathrm{~g}$ agar $\mathrm{l}^{-1}$ ) at $25^{\circ} \mathrm{C}$. For genomic DNA extraction, the fungus was grown in YPD medium (Difco) on a rotary shaker (150 r.p.m.). For RNA isolation, hyphal growth assay, and conidia germination assay, the fungus was grown in defined liquid (DL) medium, pH 6.0 (Shim \& Woloshuk, 1999). The medium $(100 \mathrm{ml})$ was inoculated with $1 \times 10^{5}$ conidia, and incubated at $25{ }^{\circ} \mathrm{C}$ shaking at 150 r.p.m., under a $14 \mathrm{~h}$ light/10 h dark cycle. For the fumonisin assay and total RNA isolation, fungal strains were grown on cracked-corn medium, as previously described (Shim \& Woloshuk, 1999). To study colony morphology and growth of fungal strains, we used diluted $(0.2 \times)$ potato dextrose agar (PDA; Difco). For macroconidia and microconidia counts, agar blocks $(0.5 \mathrm{~cm}$ diameter) were inoculated at the centre of V8 juice agar, and allowed to grow for 7 days. The conidia were harvested in $0.1 \%$ Triton X-100, and counted using a haemocytometer.

Nucleic acid isolation and manipulation. Bacterial plasmid DNA and fungal genomic DNA were extracted by using the Wizard miniprep DNA purification system (Promega) and the OmniPrep genomic DNA extraction kit (G Biosciences), respectively. Total RNA was prepared with Trizol reagent (Invitrogen), or with RNeasy plant mini kit (Qiagen). Southern and Northern analyses were performed as described previously (Sagaram et al., 2006; Sambrook \& Russell, 2001). The probes used in all hybridization experiments were labelled with ${ }^{32} \mathrm{P}$ using Prime-It II random primer labelling kit (Stratagene). DNA sequencing was performed at Gene Technologies Lab (Texas A\&M University).

PCR and quantitative real-time PCR (qRT-PCR). All primers used in this study are listed in Table 1. PCR amplifications were performed in a GeneAmp PCR system 9700 thermocycler (PE Applied Biosystems). PCR of DNA (except single-joint PCR) was performed in a $25 \mu \mathrm{l}$ total volume with Taq DNA polymerase (Promega). The PCR conditions were 2 min of pre-denaturation at $94{ }^{\circ} \mathrm{C}$, followed by 30 cycles of $45 \mathrm{~s}$ denaturation at $94{ }^{\circ} \mathrm{C}, 45 \mathrm{~s}$ annealing at $54-57{ }^{\circ} \mathrm{C}$, and 2 min extension at $72{ }^{\circ} \mathrm{C}$, unless specified otherwise. Single-joint PCR was performed using Expand Long Polymerase (Roche) using the manufacturer's suggested protocol.

qRT-PCR analyses were performed in a Cepheid Smart Cycler System with a QuantiTect SYBR Green RT-PCR kit (Qiagen). The

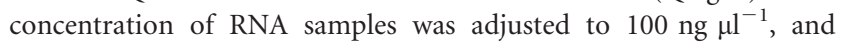
$1 \mu \mathrm{l}$ was added to the reaction mix. qRT-PCR amplifications were carried out with $30 \mathrm{~min}$ reverse transcription at $50{ }^{\circ} \mathrm{C}$, followed by 15 min pre-denaturation at $95^{\circ} \mathrm{C}$, and 35 cycles of $15 \mathrm{~s}$ denaturation at $95{ }^{\circ} \mathrm{C}, 30 \mathrm{~s}$ annealing at $55^{\circ} \mathrm{C}$, and $30 \mathrm{~s}$ extension at $72{ }^{\circ} \mathrm{C}$. The expression of $F$. verticillioides $\beta$-tubulin gene (TUB2) (GenBank accession no. U27303) was used as a reference. 
Table 1. Primers used in this study

\begin{tabular}{|c|c|c|}
\hline Number & Name & Primer sequence $\left(5^{\prime} \rightarrow 3^{\prime}\right) \dagger$ \\
\hline 1 & CPP1-che-F & CAC ATA GAC CTT CCA TTG AAG G \\
\hline 2 & HPH-R2 & CTG AAA GCA CGA GAT TCT TCG C \\
\hline 3 & CPP1-che-R & TGA TCA ATC GTC CTA ATC TCA GG \\
\hline 4 & PPE1-che-F1 & GTC ATC ACA TCC GAG GAC ATC GAG \\
\hline 5 & PPE1-com-R2 & CTG GCT CTG GCT ATT TGT TGG ATG TTG G \\
\hline 6 & CPP1-A & 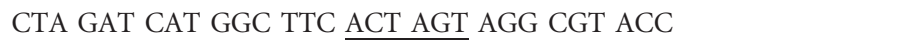 \\
\hline 7 & CPP1-B & CAC TTT GAC TTG ACT $\overline{\text { CCC GGG }}$ TGT TCA CTG \\
\hline 8 & CPP1-C & 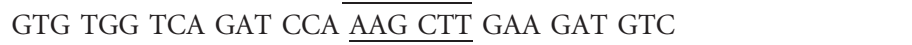 \\
\hline 9 & CPP1-D & 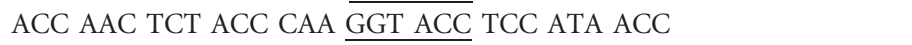 \\
\hline 13 & M13-F & TTG TAA AAC GAC GGC $\overline{\text { CAG TGA }}$ \\
\hline 14 & M13-R & CAG GAA ACA GCT ATG ACC ATG \\
\hline 15 & CPP1-LC-F & CAT GGT CAT AGC TGT TTC CTG GAT GCC TTC GCA TCC TCC CAG \\
\hline 16 & CPP1-LC-R & 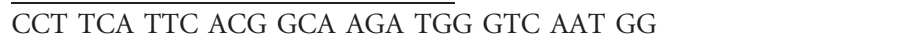 \\
\hline 17 & CPP1-LC-R2 & CGC TGT TCA AGA GTT GGG CTT TCC \\
\hline 18 & Gene-F & GCG AAT TGG AGC TCC ACC GC \\
\hline 19 & FUM1-F & ATA ACC ATC TCG GCT CAA CG \\
\hline 20 & FUM1-R2 & GTA TCT GGT GGA CAA CTT TGG G \\
\hline 21 & PKS1-F & GGA TAC ATC TCA ATG GCT GTT GAG G \\
\hline 22 & PKS1-R & CCT CAC GAA GAT TAA AGT CGT CG \\
\hline 23 & PKS2-F & GGA GTC AGT ATC AGA GTG TAG CTC \\
\hline 24 & PKS2-R & CGT CCA TAC TTT GGA GGA GAA CC \\
\hline 25 & PKS4-F & GCA TCA AGA CTA AGA TCA ACC AGG \\
\hline 26 & PKS4-R & GGC AAT GTA TGC TTC GAG AGC \\
\hline 27 & TUB2-F & CAG CGT TCC TGA GTT GAC CCA ACA G \\
\hline 28 & TUB2-R & CTG GAC GTT GCG CAT CTG ATC CTC G \\
\hline 29 & PPE1-com-F1 & CAT GGT CAT AGC TGT TTC CTG CTG TAG TCT TGT GGG CAT CG \\
\hline 30 & PPE1-com-R1 & GTG ACG ACC AGC GAA TCC CTG GTG \\
\hline 31 & RGF-F* & GAC ATC ATC GAG ACA TTA CAG GAT GC \\
\hline 32 & RGF-R* & GCT CTT CGT AAG CGA CTA GTC GG \\
\hline 33 & SepA-F* & CGA CTC CCG ATA TCA CGA CGT ACT C \\
\hline 34 & SepA-R ${ }^{*}$ & GTC GAT ACG GGT GTT ATC CTG GAC \\
\hline 35 & $\mathrm{CPO}_{-} \mathrm{F}^{*}$ & GAC AGG AAC TCC GGG AAC AAC AG \\
\hline 36 & $\mathrm{CPO}-\mathrm{R}^{*}$ & GTC CAC GGT CAT CCA TGA ATC C \\
\hline 37 & AIP-F* & CTG CCG AAG ATT CTC CCG TCA G \\
\hline 38 & AIP-R* & CAA GGT CAG ATC CTT CGT GGG AG \\
\hline
\end{tabular}

${ }^{*}$ qRT-PCR primers for F. verticillioides genes homologous to Sac. cerevisiae BNI1, BUD3, BUD6 and SPA2; the F. verticillioides genes were designated SepA, RGF, AIP and CPO, respectively.

$\dagger$ Restriction enzyme sites (underlined) were incorporated in the primers to insert amplicons into pBP15. THE M13-R primer sequence for single joint PCR application is shown in bold (Yu et al., 2004).

F. verticillioides transformation. F. verticillioides protoplasts were generated using the protocol described by Shim \& Woloshuk (2001), except that Mureinase $\left(2 \mathrm{mg} \mathrm{ml}^{-1}\right)$ was replaced with Drieselase $\left(5 \mathrm{mg} \mathrm{ml}^{-1}\right.$ ) (Sigma). The CPP1 gene disruption vector YEC2 was created by inserting $571 \mathrm{bp}$ DNA from the $5^{\prime}$ region of the CPP1 gene, and 529 bp DNA from the $3^{\prime}$ region of the CPP1 gene, into pBP15 vector, which contains a hygromycin phosphotransferase $(H P H)$ gene as a selectable marker (Fig. 1a) (Sagaram et al., 2006). Primers CPP1 A, CPP1-B, CPP1-C and CPP1-D were used to amplify the $5^{\prime}$ and $3^{\prime}$ regions, which were subsequently cloned into pBP15 vector (Fig. 1a). The vector YEC2 was linearized with NotI prior to protoplast transformation. Hygromycin-resistant transformants were selected on regeneration agar medium containing hygromycin $\left(150 \mu \mathrm{g} \mathrm{m}^{-1}\right)$, and screened for CPP1 deletion by PCR and Southern analysis. For PCR, primers (CPP1-che-F, CPP1-che-R and $\mathrm{HPH}-\mathrm{R} 2)$ that provide specific positive and negative amplification were used to detect homologous recombination events (Fig. 1a). For Southern analysis, fungal genomic DNA samples were digested with EcoRI before they were subjected to electrophoresis in a $1 \%$ agarose gel. A 500 bp DNA fragment of YEC2, excised by double enzyme digestion (SpeI and SmaI), was labelled with ${ }^{32} \mathrm{P}$ and used as a probe (Fig. 1a).

The CPP1 deletion mutant PP179 was complemented with a WT $C P P 1$ gene fused to a geneticin-resistance gene $(G E N)$. The complementation construct YEC4 was made via single-joint PCR strategy (Sagaram et al., 2006; Yu et al., 2004). Primers CPP1-LC-F and CPP1-LC-R amplified the complete CPP1 gene plus the 850 bp 5' untranslated region (UTR) and the $450 \mathrm{bp} 3^{\prime}$ UTR. This amplicon was fused with the GEN marker, and the final YEC4 construct was amplified with primers Gene-F and CPP1-LC-R2, using Expand Long Polymerase (Roche). We then transformed YEC4 into PP179 

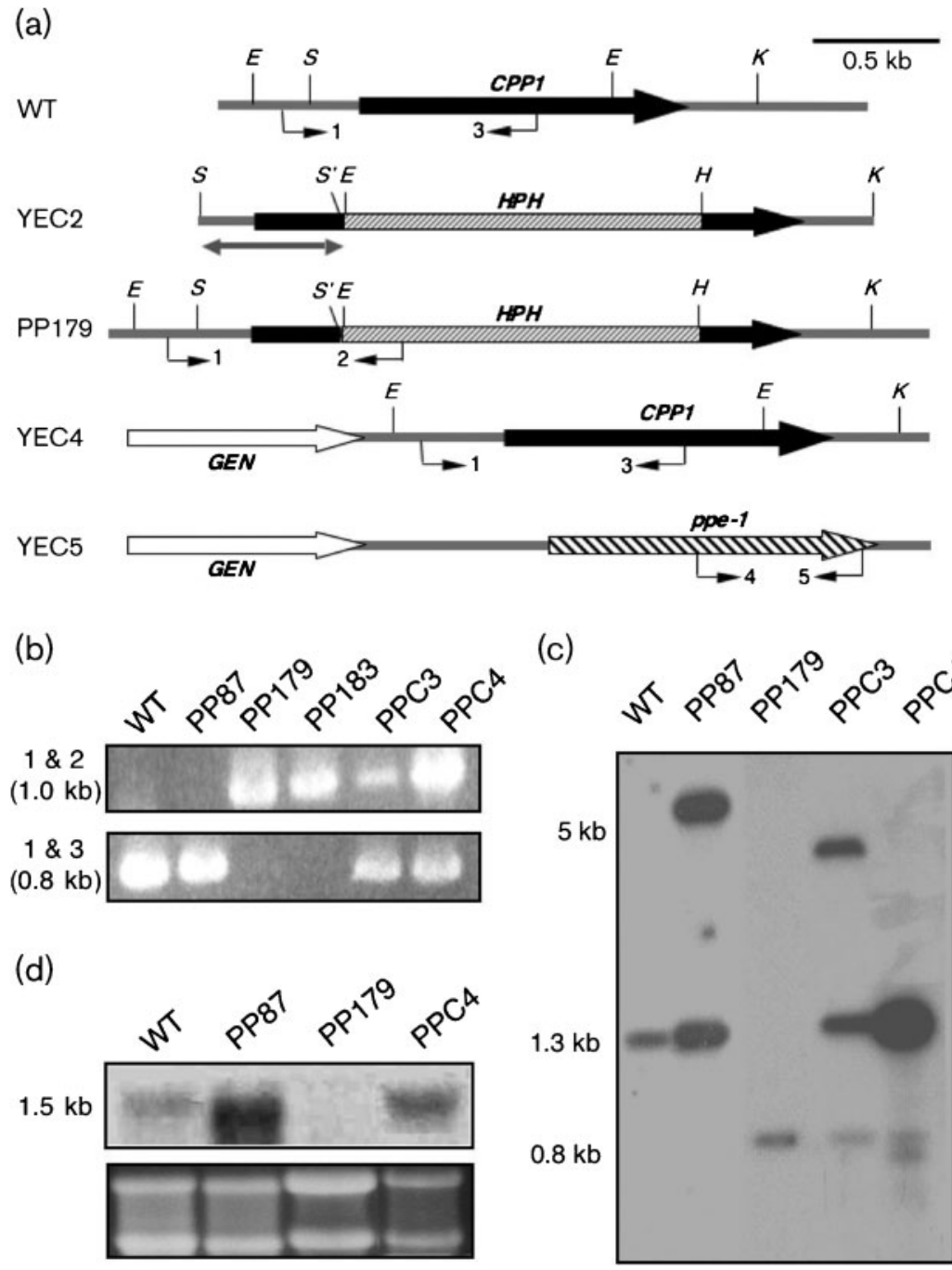

(c)
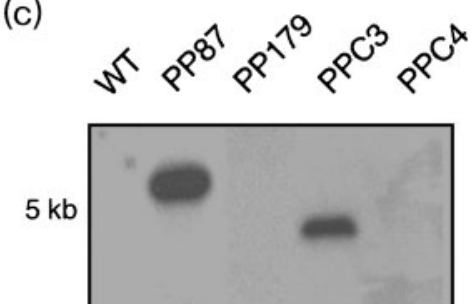

$1.3 \mathrm{~kb}$

$0.8 \mathrm{~kb}$
Fig. 1. Molecular characterization of F. verticillioides CPP1. (a) WT, schematic representation of the CPP1 locus in the WT strain. YEC2, the CPP1 disruption construct, which harbours $H P H$ as the selectable marker. PP179, schematic representation of CPP1 locus in the knockout strain after the homologous recombination event. YEC4, the construct with CPP1 WT gene fused to GEN that was used to complement PP179. YEC5, the construct with $N$. crassa ppe-1 gene fused to GEN that was used to complement PP179. The left-right arrow on YEC2 indicates the fragment used as the ${ }^{32} \mathrm{P}$-labelled probe in the Southern blot. The numbered arrows indicate the location of primers used for positive and negative PCR assays (Table 1). S, Spel; S', Smal; H, Hindlll; K, Kpnl. (b) PCR analysis of CPP1 disruption and complementation using the primers described in (a). PP87, ectopic integration strain; PP179 and PP183, CPP1 knockout strains; PPC3, YEC3-complemented strain (for information on PPC3, see supplementary data, section 1 , available with the online version of this paper); PPC4, YEC4complemented strain. The numbers on the left indicate primer combinations for PCR amplification. (c) Southern blot analysis of transformants. Fungal genomic DNA was digested with EcoRl, and the blot was hybridized with ${ }^{32} \mathrm{P}$-labelled DNA probe [shown in (a)]. Molecular sizes are indicated on the left. (d) Northern blot analysis of the transformants probed with ${ }^{32} \mathrm{P}$-labelled DNA fragment [shown in (a)] to determine the expression of CPP1. Total RNA samples $(15 \mu \mathrm{g})$ were subjected to electrophoresis in a $1.2 \%$ denaturing agarose gel. The gel was stained with ethidium bromide to confirm uniformity of loading (rRNA). protoplasts, and screened for colonies resistant to geneticin and hygromycin (Sagaram et al., 2006). The introduction of YEC4 in geneticin-resistant strains was verified by PCR and Southern blot analyses.

Fumonisin $\mathbf{B}_{\mathbf{1}}$ analysis. $F$. verticillioides strains were grown on cracked-corn medium for 10 days for fumonisin production analysis. $\mathrm{FB}_{1}$, the major fumonisin produced by $F$. verticillioides, was extracted and analysed by HPLC, following the method described by Shim \& Woloshuk (1999). In addition to $\mathrm{FB}_{1}$ HPLC analysis, qRT-PCR was used to investigate the relative expression of FUM1 and other selected PKS genes (Kroken et al., 2003) in the WT and mutant strains. Total RNA samples were prepared from the WT, PP87, PP179 and PPC4 grown on cracked-corn medium for 10 days, and qRT-PCR analysis was performed with SYBR-Green as the fluorescent reporter, using gene-specific primers (FUM1-F and FUM1-R2) for the FUM1 gene. The expression of each gene was normalized to endogenous TUB2 gene expression. The gene expression was calibrated using $2^{-\Delta \Delta C_{\mathrm{T}}}$ method ( $C_{\mathrm{t}}$, threshold cycle) (Livak \& Schmittgen, 2001); the range of expression was calibrated using $2^{-\Delta \Delta C_{\mathrm{T}}-s}-2^{-\Delta \Delta C_{\mathrm{T}}+s}$, where $s$ is the standard deviation of the $\Delta C_{\mathrm{t}}$ value. Subsequently, the total RNA samples of the WT and PP179 strains were subjected to qRT-PCR analysis using gene-specific primers for FUM1, PKS1 (PKS1-F and PKS1-R), PKS2 (PKS2-F and PKS2-R), and PKS4 (PKS4-F and PKS4$\mathrm{R})$. Again, the gene expression was calibrated using the $2^{-\Delta \Delta C_{\mathrm{T}}}$ method, with TUB2 as the endogenous control.

Microscopy. Microscopic observations were made using an Olympus BX51 microscope (Olympus America). A detailed description of features used for imaging from this microscope has been given (Shaw \& Upadhyay, 2005). Imaging of hyphal growth phenotypes was performed using an Olympus DP70 camera and DP70-BSW software (version 01.01). Nuclei were stained with Hoechst 33258 dye, as described previously (Shaw \& Upadhyay, 2005).

Complementation of PP179 strain with $\mathbf{N}$. crassa ppe-1. $\mathrm{N}$. crassa ppe-1 (GenBank accession no. XM_951629) encodes a probable cell-shape-control protein phosphatase. The complementation construct YEC5 was generated by single-joint PCR strategy (Fig. 1a) (Yu et al., 2004). WT ppe-1 (1.43 kb ppe-1 gene plus $1.6 \mathrm{~kb} 5^{\prime}$ UTR and 
500 bp 3' UTR) was amplified from the $N$. crassa genomic DNA using the primers PPE1-com-F1 and PPE1-com-R1. These amplicons were fused to GEN by joint-PCR using PPE1-com-R2 and Gene-F primers. The final construct, YEC5 was introduced into the protoplasts of PP179, and the transformants were screened for geneticin resistance. The selected isolates were further analysed by PCR, using primers PPE1-che-F1 and PPE1-com-R2 to determine the presence of YEC5 in the genome.

\section{RESULTS}

\section{F. verticillioides CPP1 encodes a putative protein phosphatase 2A catalytic subunit}

The F. verticillioides gene index (The Dana Farber Cancer Institute, http://compbio.dfci.harvard.edu/tgi/fungi.html) and Fusarium group database (Broad Institute of Harvard and MIT, http://www.broad.mit.edu/annotation/fgi/) were screened using the $300 \mathrm{bp}$ EST sequence ( $\mathrm{ft536}$ _ 0_M14) for matching cDNA and genomic DNA sequences. The gene index screen resulted in the identification of a 1468 bp tentative consensus 26726, which contains a $1221 \mathrm{bp}$ ORF. The Fusarium group database search revealed that supercontig 13 in chromosome 1, specifically sequence 301853 to 303473 (FVEG_09543), harbours a matching genomic DNA sequence. Taken together, in silico analysis revealed that the CPP1 gene is $1377 \mathrm{bp}$ in length, contains three introns $(53,51$ and $49 \mathrm{bp})$, and is predicted to encode a 407 aa polypeptide.

Sequence analysis of Cpp1 revealed a type 2A protein phosphatase catalytic domain (PP2Ac) between amino acids 32 and 385 . This domain is present among a large family of serine/threonine phosphatases. Likewise, Cpp1 displays a high similarity to the PP2Ac domains in a number of eukaryotes, particularly those present in filamentous fungi (Fig. 2). Namely, Cpp1 shares significant similarity with PPE-1 protein, which is a probable cell-shape-control PP2Ac in $N$. crassa ( $E$ value, 0.0 ) (unpublished; GenBank accession no. XM_951629), and with SitA, which is a PP2Ac involved in the TOR pathway of Aspergillus nidulans ( $E$ value, 8e-165) (Fitzgibbon et al., 2005; GenBank accession no. CAG30555). Significantly, these PP2Ac proteins of filamentous fungi are orthologous to the Schizosaccharomyces pombe protein Ppe1, which plays a role in cell morphogenesis and mitosis (Shimanuki et al., 1993). We determined via Northern blot analysis that there is no significant differential expression of CPP1 during the course of fungal growth in DL culture, thereby concluding that CPP1 is a constitutively expressed gene in F. verticillioides (data not shown).

\section{Deletion of CPP1 results in upregulation of FUM1 and overproduction of $\mathrm{FB}_{\mathbf{1}}$}

$F$. verticillioides $C P P 1$ gene knockout strains were generated using a double homologous recombination strategy in order to test our hypothesis that CPP1 is associated with $\mathrm{FB}_{1}$ biosynthesis. Of the 243 hygromycin-resistant transformants, two strains, designated PP179 and PP183, were identified in which CPP1 was replaced by the YEC2 construct. Specific primers, designed to produce a PCR amplicon dependent upon homologous recombination, were used to confirm the homologous recombination event. PP179 and PP183 produced the expected band; however, no amplicon was observed for the WT or strain PP87, which is a strain with ectopic YEC2 integration (Fig. 1b). We selected PP179 for further molecular characterization. Southern blot analysis further confirmed that the $H P H$ gene replaced 419 bp DNA within the CPP1 gene. The $571 \mathrm{bp}{ }^{32} \mathrm{P}$-labelled DNA probe hybridized to a $1.3 \mathrm{~kb}$ band in the WT and PP87 strains, whereas it hybridized to a $0.8 \mathrm{~kb}$ band in the PP179 strain (Fig. 1c). Northern blot analysis was performed using a $571 \mathrm{bp} \mathrm{CPP1}$ DNA fragment probe, which hybridized to the expected

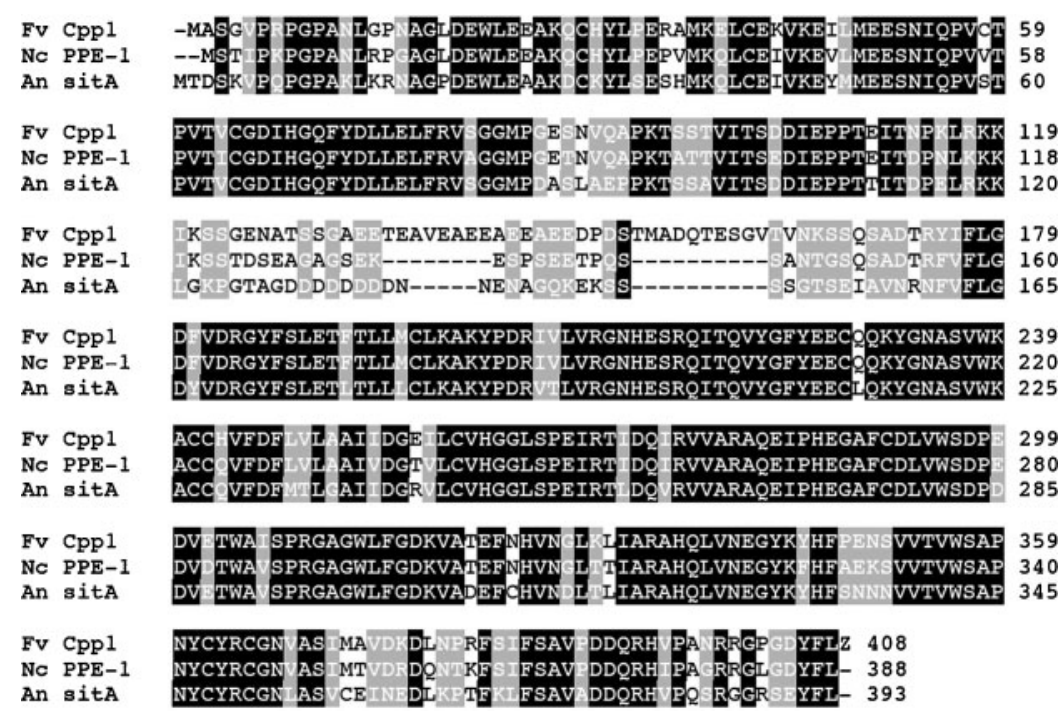

Fig. 2. Amino acid alignment of $F$. verticillioides Cpp1, N. crassa PPE-1 and Asp. nidulans SitA via CLUSTALW. Amino acids common to all proteins are indicated by white letters on a black background. Similar amino acids are indicated by white letters on a grey background. Gaps introduced for alignment are indicated by dashes. Cpp1 protein shares significant similarity with PPE-1 (80\% identity and $87 \%$ similarity; $E$ value, 0.0$)$ and SitA (70\% identity and $78 \%$ similarity at the amino acid level; $E$ value, 8e-165). 
$1.35 \mathrm{~kb}$ transcript band in the WT and PP87, but did not hybridize in PP179 (Fig. 1d).

Measurement of the radial growth of $F$. verticillioides strains on $0.2 \times$ PDA plates revealed that PP179 had approximately a $40 \%$ reduction in growth rate when compared with the WT progenitor and the PP87 strain (Fig. 3). However, no significant difference in fungal mass (fresh wet weight) was observed when strains were grown in $0.2 \times$ PDB and YPD broth. Subsequently WT, PP87 and PP179 strains were grown on cracked-corn medium and $\mathrm{DL}$ medium to test $\mathrm{FB}_{1}$ production. TLC and HPLC analysis revealed that the WT and PP87 did not differ in $\mathrm{FB}_{1}$ production in either cracked-corn medium or $\mathrm{DL}$ medium; however, we observed a significant increase in $\mathrm{FB}_{1}$ production in $\mathrm{PP} 179$. Specifically, $\mathrm{FB}_{1}$ production on inoculated cracked-corn medium was more than four times greater for PP179 than for the WT (Fig. 4a).

To verify that the growth phenotype and increased $\mathrm{FB}_{1}$ production of PP179 were due to the deletion of CPP1, PP179 was complemented using YEC4, which contains a WT copy of the CPP1 gene fused to GEN (Fig. 1a). Following transformation, complemented strains were selected by testing growth on $0.2 \times \mathrm{PDA}$ containing

(a)

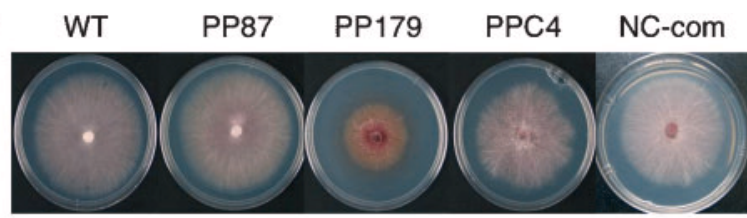

(b)

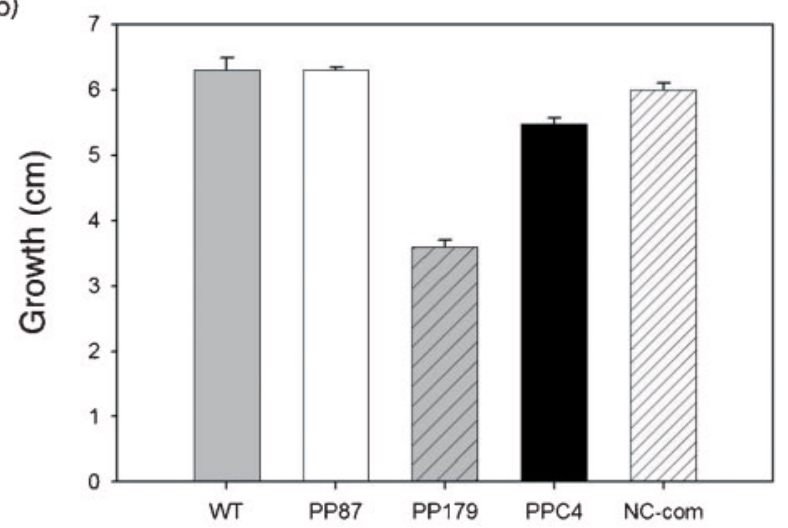

Fig. 3. Colony morphology and growth of the WT and mutants grown on $0.2 \times$ PDA. (a) Strains were point inoculated with an agar block ( $0.5 \mathrm{~cm}$ diameter), and incubated for 7 days at $25^{\circ} \mathrm{C}$, under a $14 \mathrm{~h}$ light/10 h dark cycle. PP87, ectopic integration strain; PP179, CPP1 knockout strains; PPC4, YEC4-complemented strain; NC-com, YEC5-complemented strain. Growth was significantly impaired in PP179, whereas in PPC4 and NC-com strains, the growth defect was restored to that of the WT progenitor. (b) Radial growth measured and presented as a bar graph. Results are means \pm SD of three biological replications. hygromycin and geneticin. The putative complemented strain containing YEC4 was designated PPC4. We determined via PCR that PPC4 contained the introduced CPP1 gene (from YEC4) and the HYG-replaced mutant locus (Fig. 1b). Southern analysis verified the PCR data (Fig. 1c). Northern blot analysis of PPC4 showed that CPP1 gene expression was restored in PPC4 (Fig. 1d). Growth rate reduction and increased $\mathrm{FB}_{1}$ reduction in PP179 were restored to the level of the WT in the PPC4 strain, thus providing further evidence for roles of $C P P 1$ in growth rate and $\mathrm{FB}_{1}$ production (Fig. 3 and $4 \mathrm{a}$ ).

\section{Increased $\mathrm{FB}_{1}$ production in PP179 is due to upregulation of FUM1, the $\mathrm{FB}_{1}$-specific PKS gene}

FUM1 encodes a PKS that is critical to $\mathrm{FB}_{1}$ biosynthesis (Proctor et al., 1999). Here, we asked the question whether the drastic increase in $\mathrm{FB}_{1}$ production in $\mathrm{PP} 179$ is due to altered FUM1 expression. Total RNA was harvested from WT, PP87, PP179 and PPC4 strains grown on cracked-corn medium, the culture condition used for $\mathrm{FB}_{1}$ production. qRT-PCR analysis of FUM1 revealed at least 11-fold higher FUM1 expression in PP179 than in other strains tested (Fig. 4b). Analysis of variance (ANOVA) of the gene expression data from all four strains $(P<0.01)$ suggested that FUM1 expression levels in the WT, PP87 and PPC4 strains were significantly different from that of PP179 strain. When the test was limited to the WT, PP87 and PPC4, the resulting $P$ value was 0.862 , suggesting that the complementation of PP179 with the CPP1 gene restored the WT level of FUM1 expression. The data provide molecular evidence that $C P P 1$ serves as a negative regulator of $\mathrm{FB}_{1}$ biosynthetic genes in $F$. verticillioides.

As there are over 15 PKS genes in F. verticillioides (Kroken et al., 2003), we next investigated the specificity of CPP1 to regulation of FUM1. Kroken et al. (2003) classified 15 typeI PKS genes present in F. verticillioides into major clades or subclades. To test the impact of CPP1 deletion on the expression of PKS genes, we selected four PKS genes from different subclades: PKS1 (reducing PKS clade II), PKS2 (reducing PKS clade I), PKS4 (non-reducing PKS clade I) and PKS11 (FUM1, reducing PKS clade III). qRT-PCR analysis of the four PKS genes revealed that only FUM1 expression was upregulated significantly in PP179, whereas $P K S 1, P K S 2$ and PKS4 were either downregulated or unchanged (Fig. 4c). The $t$ tests for PKS1, PKS2 and PKS4 expression confirmed no significant difference in expression between the WT and PP179. Our results showed that the major impact of CPP1 deletion was on FUM1 expression, suggesting that CPP1 may have a specific function to regulate FUM1 in F. verticillioides.

\section{CPP1 is required for hyphal polarity maintenance in $\boldsymbol{F}$. verticillioides}

In contrast to the WT and PP87, PP179 showed a distinguishable hyphal swelling when grown in liquid 

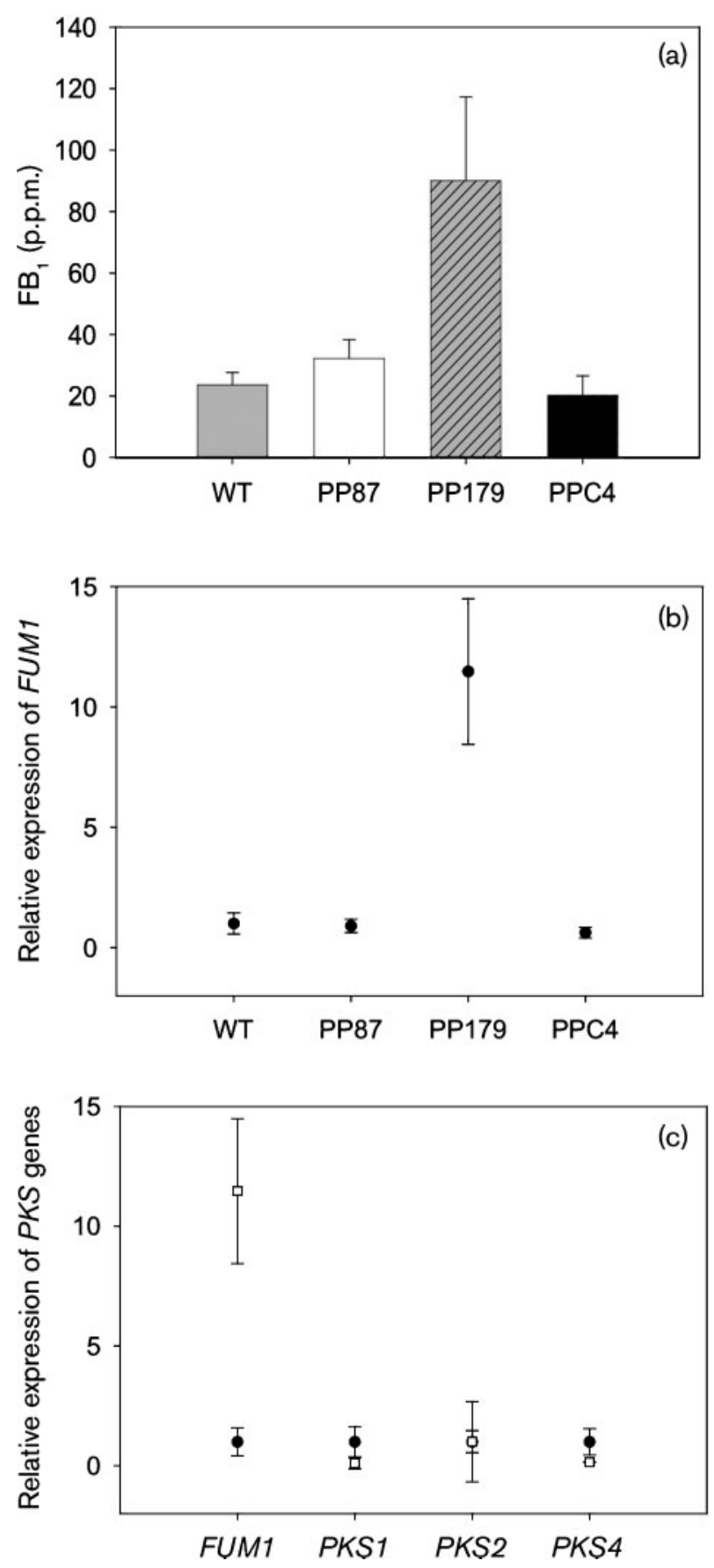

medium (Fig. 5a). This phenotype in PP179 became more apparent in the cultures incubated longer than 7 days (data not shown). Nucleus staining was utilized to investigate whether the hyphal-swelling phenotype in PP179 was linked to defective cell-cycle progression. Swollen hyphae of PP179 contained multiple nuclei, whereas nuclei in the WT were uniformly distributed along hyphae (Fig. 5b). This phenotype of PP179 suggested that cell-cycle progression was not affected, but maintenance of hyphal polarity was impaired. Notably, suppression of hyphal swelling in
Fig. 4. $\mathrm{FB}_{1}$ analysis, and $F U M 1$ expression. (a) Quantification of $\mathrm{FB}_{1}$ production in WT, PP87, PP179 and PPC4 strains. Sterile cracked corn $(2 \mathrm{~g})$ was inoculated with an agar block $(0.5 \mathrm{~cm}$ diameter) of WT and mutant strains. After incubation for 10 days at $25{ }^{\circ} \mathrm{C}, \mathrm{FB}_{1}$ was extracted with $10 \mathrm{ml} 50 \%$ acetonitrile in water, purified through SPE C18 columns, eluted in $2 \mathrm{ml} 70 \%$ acetonitrile in water, and quantified by HPLC. All values represent means (SD) of three biological replications. (b) Expression of FUM1 gene in WT, PP87, PP179 and PPC4 strains. Total RNA samples were prepared from fungal strains grown in cracked-corn medium for 10 days, and quantitative real-time PCR analysis was performed with SYBR-Green as the fluorescent reporter. The levels of transcription were evaluated using the $2^{-\Delta \Delta C_{T}}$ method, with TUB2 as the endogenous control. Data represent the fold differences in gene expression. Three biological replications were performed to obtain standard deviations. (c) Expression analysis of select PKS genes in the WT and PP179. Total RNA samples were prepared from fungal strains grown in cracked-corn medium for 10 days, and qRT-PCR analysis was performed with SYBR-Green as the fluorescent reporter. The levels of transcription were evaluated using the $2^{-\Delta \Delta C_{T}}$ method, with TUB2 as the endogenous control. Data represent the fold differences in gene expression. Three biological replications were performed to obtain standard deviations. - WT; $\square$, PP179.

PP179 occurred with the addition of sucrose (1 M) to the medium (Fig. 5c), and this suppression was maintained for up to 10 days. PPC4 showed complete recovery from hyphal swelling demonstrating that this phenotype was due to CPP1 deletion (Fig. 5a).

We next identified well-characterized Saccharomyces cerevisiae polarity genes BNI1, BUD3, BUD6 and SPA2 (Amberg et al., 1997; Chant et al., 1995; Sagot et al., 2002; Sheu et al., 1998). Homologues of these genes in filamentous fungi are known to be associated with cellpolarity maintenance and cytokinesis (Harris et al., 1997; Virag \& Harris, 2006; Wendland, 2003). We isolated corresponding F. verticillioides homologues FVEG_04885.3, FVEG_06243.3, FVEG_11303.3 and FVEG_00700.3, and tested the transcriptional levels in the WT and PP179 strains. qRT-PCR, using gene-specific primers (Table 1), showed no significant difference between the WT and PP179 in expression levels of these genes (data not shown).

\section{CPP1 is involved in microconidia-macroconidia equilibrium and conidia germination in $\boldsymbol{F}$. verticillioides}

One striking feature of PP179 was the high percentage of macroconidia production on $\mathrm{V} 8$ agar medium. In $F$. verticillioides, the formation of macroconidia has been shown under a few select conditions, such as UV exposure (Nelson et al., 1983). The WT and PP87 strains produced microconidia, but not macroconidia, on V8 agar. In contrast, approximately $44 \%$ of the conidia harvested from PP179 on V8 agar were macroconidia (Fig. 6a). The conidiation profile was the same as the WT in PPC4. We 
(a)

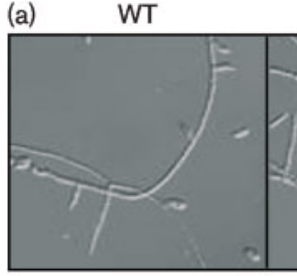

PP87

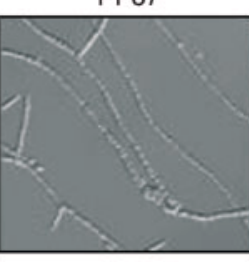

(b)

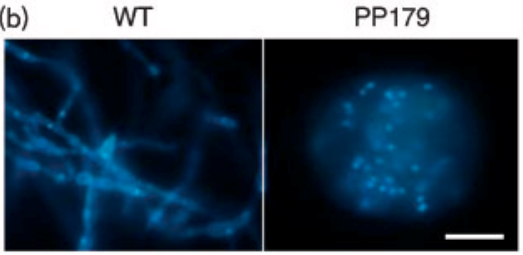

(d)

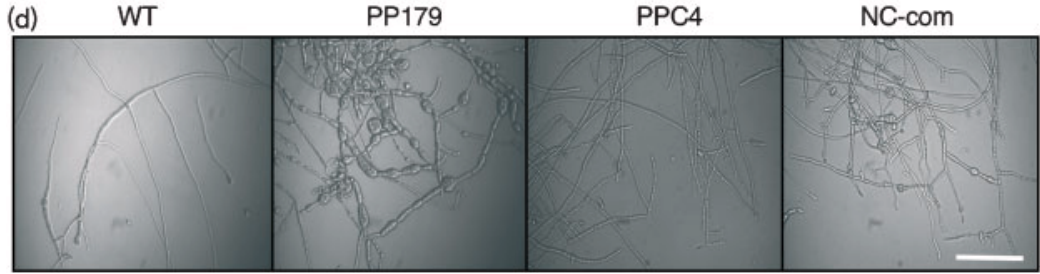

(c) WT + sucrose

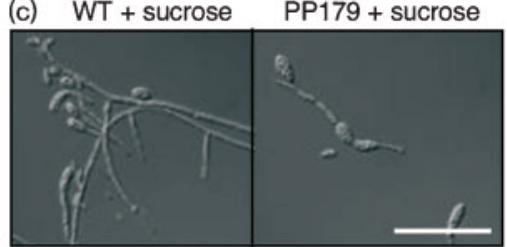

PPC4

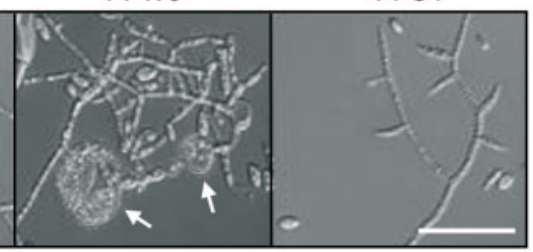

PP179 + sucrose

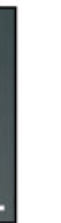


with 5' and 3' UTR (Fig. 1a), was transformed into PP179 protoplasts, and the transformants were selected by geneticin and hygromycin resistance. Isolates were further tested by PCR to demonstrate the presence of WT ppe-1. No amplicon of ppe-1 was detected in the WT and PP179 strains, whereas amplicons of the expected size $(1.5 \mathrm{~kb})$ were observed in the YEC5-complemented isolates (data not shown). One isolate was selected, and designated 'NCcom' strain, and further analysed. Interestingly, the complementation of PP179 with YEC5 resulted in restorations of WT morphology, and the reduced growth rate of PP179 was restored to WT level in NC-com (Fig. 3). Notably, the hyphal swelling phenotypes of PP179 were less apparent in NC-com (Fig. 5d). Macroconidia production by PP179 was completely eliminated in NC-com, and germination efficiency was restored to that of WT progenitor (data not shown). Elevated $\mathrm{FB}_{1}$ production in PP179 was significantly reduced in NC-com similar to the WT level (data not shown). These results strongly support the hypothesis that $N$. crassa PPE-1 and F. verticillioides Cpp1 are functionally conserved across the two species.

\section{DISCUSSION}

In eukaryotic cells, reversible protein phosphorylation provides an important means to regulate various cellular functions in response to external signals. While protein kinases take part in phosphorylation, protein phosphatases catalyse the dephosphorylation of proteins, thereby exerting a fundamental role in regulating cellular processes (Dickman \& Yarden, 1999). It has been suggested that about one-third of the eukaryotic proteins are regulated by reversible phosphorylations of specific serine, threonine and/or tyrosine residues (Ceulemans \& Bollen, 2004). To date, the major group of serine/threonine protein phosphatases are encompassed by two different structural families. PP1, PP2A, PP2B and PP5 are classified as members of the protein phosphatase P family, while PP2C is a member of $\mathrm{Mg}^{2+}$-dependent protein phosphatase $\mathrm{M}$ family (Dickman \& Yarden, 1999). Each protein phosphatase has specific or overlapping roles in regards to regulating cellular functions. Despite their central roles in regulating a wide variety of cellular function, including signal transduction and gene expression, limited information is available on the functions of protein phosphatases in filamentous fungi. In general, fungi have a larger number of protein kinases than protein phosphatases in their genome. For instance, Sac. cerevisiae has approximately 6000 genes, of which 113 encode protein kinase genes, but only 31 encode protein phosphatases (Dickman \& Yarden, 1999; Sakumoto et al., 1999; Stark, 1996). Considering the deviation in prevalence between protein kinases and protein phosphatases, it is likely that Cpp1 has broad substrate specificity, and interacts with multiple protein kinases in several signalling pathways. Further complicating the process, a $36 \mathrm{kDa}$ PP2A catalytic subunit combines with a $65 \mathrm{kDa}$ regulatory subunit to form a PP2A holoenzyme dimer, with a third variable subunit, known as the B subunit, responsible for substrate specificity (Stark, 1996; Mayer-Jaekel \& Hemmings, 1994). Since CPP1 encodes a putative catalytic subunit of $\mathrm{PP} 2 \mathrm{~A}$, the broad specificity of substrates of CPP1 may be narrowed with additional protein phosphatase subunit components (Mayer-Jaekel \& Hemmings, 1994).

In this study, our main hypothesis was that $C P P 1$ serves as a putative $\mathrm{FB}_{1}$ regulatory gene, perhaps in a negative or suppressive manner. Consistent with our hypothesis, we detected elevated $\mathrm{FB}_{1}$ production in PP179 via TLC and HPLC analyses (Fig. 4a), and subsequent qRT-PCR analysis suggested that this increased $\mathrm{FB}_{1}$ production is a result of an elevated FUM1 expression (Fig. 4b). PKS1 and PKS2 were classified, along with FUM1, as reducing PKSs, whereas PKS4 resided in a separate group of non-reducing PKSs (Kroken et al., 2003). Interestingly, deletion of CPP1 did not have a drastic effect on the expression of other PKS genes, suggesting that the regulatory role of $C P P 1$ is specific to FUM genes and $\mathrm{FB}_{1}$ biosynthesis (Fig. 4c). An expanded transcriptional profiling study is necessary to help us better understand the role of CPP1 in F. verticillioides secondary metabolism pathways. Of particular interest is whether CPP1 affects other fumonisin biosynthesis regulatory genes, particularly the genes FUM21, ZFR1 and GBB1 that are positively associated with fumonisin biosynthesis (Brown et al., 2007; Flaherty \& Woloshuk, 2004; Sagaram \& Shim, 2007). Meanwhile, it is well recognized that type $2 \mathrm{~A}$ protein phosphatases are associated with cell differentiation and development in eukaryotes, including filamentous fungi (Dickman \& Yarden, 1999; Hunter, 1995; Mayer-Jaekel \& Hemmings, 1994; Shenolikar, 1994; Yatzkan et al., 1998), and multiple PP179 phenotypes suggested that CPP1 is also involved in multiple downstream signalling pathways in F. verticillioides. Strikingly, CPP1 deletion led to a hyphal-swelling phenotype, thereby suggesting the functional role of CPP1 in morphogenesis. We hypothesized that hyphal swelling in PP179 is caused by random deposition of cell wall components, leading to impaired hyphal polarity. In the yeast Sac. cerevisiae, mutation in genes BNI1, BUD3, BUD6 and SPA2, which regulate polarity and proper cell development, triggers cellpolarity phenotypes (Amberg et al., 1997; Chant et al., 1995; Sagot et al., 2002; Sheu et al., 1998). While there is no experimental evidence that homologues of these yeast genes are involved in F. verticillioides cell-polarity maintenance, studies in other filamentous fungi provide support to our hypothesis. For instance Asp. nidulans sepA, the $B N I 1$ homologue, plays a critical role in cytokinesis, and is required for maintenance of polarity during hyphal growth (Harris et al., 1997). In Ashbya gossypii, deletion of $A g B U D 3$, the BUD3 homologue, led to a change in actin ring localization, and subsequent aberrant chitin accumulation, ultimately generating delocalized septa (Wendland, 2003). However, our results suggest that hyphal swelling in PP179 is not due to transcriptional defect in F. verticillioides BNI1, BUD3, BUD6 and 
SPA2-like genes. We are currently investigating putative signalling pathways downstream of CPP1 involved in maintaining hyphal polarity and growth in F. verticillioides.

In Sac. cerevisiae, PP2A-related genes have been identified, and designated PPH21, PPH22, PPH3, SIT4 and PPG1 (Zabrocki et al., 2002). A phylogenic tree containing the well-characterized protein phosphatases in Sac. cerevisiae clearly indicates that Cpp1 has the highest homology with Sit4 (76\% identity, $84 \%$ similarity). Sit4 is a component of the conserved TOR (the target of rapamycin) signalling pathway (Di Como \& Arndt, 1996) that interacts with Tap42, the phosphatase regulatory subunit of the TOR pathway, in response to nutrient stress (Cutler et al., 2001). Considering the similarity between Sit4 and Cpp1, we hypothesized that hyphal swelling in PP179 may be caused by the defect in nutritional stress response, and perhaps this process is mediated by $F$. verticillioides Tap42 homologue (FVEG_06413.3) (Fig. 5a, b). The hyphal swelling phenotype in PP179 was particularly apparent in the later stages of growth, perhaps when the fungus is under nutritional stress. In contrast, the hyphal swelling could be reversed in PP179 by osmotic remediation with sucrose (Fig. 5c). In Sac. cerevisiae, the protein phosphatase catalytic subunit Sit4 is implicated in $G_{1}$ to $S$ transition (Jiang, 2006), and this leads us to presume that growth and germination defects in PP179 are the consequence of a defect in cell-cycle progression (Fig. 3 and 6b). In Asp. nidulans, an amino acid change in PP2A protein (PphA) led to a growth defect, lack of germ tube, and mitotic defect (Kosmidou et al., 2001). In N. crassa, the transcript levels of $p p p-1$ were increased during hyphal germination and elongation (Zeke et al., 2003). We also speculate that the germination defect in PP179 may be linked to hyphal swelling. The hyphal polarity defect caused by CPP1 deletion may have initially affected germination, and later led to hyphal swelling.

A report by Yatzkan \& Yarden (1999) has suggested a link between PP2A regulatory subunit $r g b-1$ and macroconidiation in $N$. crassa: inactivation of $r g b-1$ leads to a failure in the formation of mature macroconidia. In contrast, we observed that macroconidiation was activated in PP179 (Fig. 6a). Since macroconidia production occurs rarely in F. verticillioides under laboratory conditions (Nelson et al., 1983), it is reasonable to presume that CPP1 plays a role in suppressing macroconidia production in the WT strain. We identified the homologue of $r b g-1$ in $F$. verticillioides genome (FVEG_01508.3), and we are currently investigating its role. Moreover, recent characterization of $F$. verticillioides FvVE1 revealed that deletion of this gene results in macroconidiation and growth defect phenotypes similar to those of PP179 (Li et al., 2006). However, the molecular genetic linkage between CPP1 and FvVE1 has not been determined to date. Taken as a whole, we anticipate that additional proteins, such as other protein phosphatase subunit components and putative Cpp1interacting proteins, are involved in regulating secondary metabolism and fungal development in F. verticillioides.
Identification and functional characterization of these proteins will help us unravel the complex regulatory mechanisms associated with Cpp1.

\section{ACKNOWLEDGEMENTS}

We thank Dr Brian D. Shaw for providing expertise on microscopic imaging, and careful reading of this manuscript. Financial support was provided by the USDA-NRI Food Safety Program (2005-3520116233) and USDA-NRI Interagency Microbial Genome Sequencing Program (2005-35600-16405).

\section{REFERENCES}

Amberg, D. C., Zahner, J. E., Mulholland, J. W., Pringle, J. R. \& Botstein, D. (1997). Aip3p/Bud6p, a yeast actin-interacting protein that is involved in morphogenesis and the selection of bipolar budding sites. Mol Biol Cell 8, 729-753.

Brown, D. W., Butchko, R. A., Busman, M. \& Proctor, R. (2007). The Fusarium verticillioides FUM gene cluster encodes a $\mathrm{Zn}$ (II)2Cys6 protein that affects FUM gene expression and fumonisin production. Eukaryot Cell 6, 1210-1218.

Ceulemans, H. \& Bollen, M. (2004). Functional diversity of protein phosphatase-1, a cellular economizer and reset button. Physiol Rev 84, $1-39$.

Chant, J., Mischke, M., Mitchell, E., Herskowitz, I. \& Pringle, J. R. (1995). Role of Bud3p in producing the axial budding pattern of yeast. J Cell Biol 129, 767-778.

Cutler, N. S., Pan, X., Heitman, J. \& Cardenas, M. E. (2001). The TOR signal transduction cascade controls cellular differentiation in response to nutrients. Mol Biol Cell 12, 4103-4113.

Di Como, C. J. \& Arndt, K. T. (1996). Nutrients, via the Tor proteins, stimulate the association of Tap42 with type 2A phophatases. Genes Dev 10, 1904-1916.

Dickman, M. B. \& Yarden, O. (1999). Serine/threonine protein kinases and phosphatases in filamentous fungi. Fungal Genet Biol 26, 99-117.

Fitzgibbon, G. J., Morozov, I. Y., Jones, M. G. \& Caddick, M. X. (2005). Genetic analysis of the TOR pathway in Aspergillus nidulans. Eukaryot Cell 4, 1595-1598.

Flaherty, J. E. \& Woloshuk, C. P. (2004). Regulation of fumonisin biosynthesis in Fusarium verticillioides by a zinc binuclear cluster-type gene, ZFR1. Appl Environ Microbiol 70, 2653-2659.

Flaherty, J. E., Pirttila, A. M., Bluhm, B. H. \& Woloshuk, C. P. (2003). PAC1, a pH-regulatory gene from Fusarium verticillioides. Appl Environ Microbiol 69, 5222-5227.

Gelderblom, W. C. A., Jaskiewicz, K., Marasas, W. F. O., Thiel, P. G., Horak, R. M., Vleggaar, R. \& Kriek, N. P. J. (1988). Fumonisins - novel mycotoxins with cancer-promoting activity produced by Fusarium moniliforme. Appl Environ Microbiol 54, 1806-1811.

Harris, S. D., Hamer, L., Sharpless, K. E. \& Hamer, J. E. (1997). The Aspergillus nidulans sepA gene encodes an $\mathrm{FH} 1 / 2$ protein involved in cytokinesis and the maintenance of cellular polarity. EMBO J 16, 3474-3483.

Hunter, T. (1995). Protein kinases and phosphatases: the yin and yang of protein phosphorylation and signaling. Cell 80, 225-236.

Jiang, Y. (2006). Regulation of the cell cycle by protein phosphatase 2A in Saccharomyces cerevisiae. Microbiol Mol Biol Rev 70, 440-449.

Kosmidou, E., Lunness, P. \& Doonan, J. H. (2001). A type 2A protein phosphatase gene from Aspergillus nidulans is involved in hyphal morphogenesis. Curr Genet 39, 25-34. 
Kroken, S., Glass, N. L., Taylor, J. W., Yoder, O. C. \& Turgeon, B. G. (2003). Phylogenomic analysis of type I polyketide synthase genes in pathogenic and saprobic ascomycetes. Proc Natl Acad Sci U S A 100, 15670-15675.

Li, S., Myung, K., Guse, D., Donkin, B., Proctor, R. H., Grayburn, W. S. \& Calvo, A. M. (2006). FvVE1 regulates filamentous growth, the ratio of microconidia to macroconidia and cell wall formation in Fusarium verticillioides. Mol Microbiol 62, 1418-1432.

Livak, K. J. \& Schmittgen, T. D. (2001). Analysis of relative gene expression data using real-time quantitative PCR and the $2^{-\Delta \Delta C_{\mathrm{T}}}$ method. Methods 25, 402-408.

Marasas, W. F. O. (2001). Discovery and occurrence of the fumonisins: a historical perspective. Environ Health Perspect 109, 239-243.

Mayer-Jaekel, R. E. \& Hemmings, B. A. (1994). Protein phosphatase 2A - a 'ménage à trois'. Trends Cell Biol 4, 287-291.

Minorsky, P. V. (2002). The hot and the classic. Plant Physiol 129, 5-6. Missmer, S. A., Suarez, L., Felkner, M., Wang, E., Merrill, A. H., Jr, Rothman, K. J. \& Hendricks, K. A. (2006). Exposure to fumonisins and the occurrence of neural tube defects along the Texas-Mexico border. Environ Health Perspect 114, 237-241.

Nelson, P. E., Marasas, W. F. O. \& Toussoun, T. A. (1983). Fusarium Species; an Illustrated Manual for Identification. University Park, PA: The Pennsylvania State University Press.

Park, D. L. \& Troxell, T. C. (2002). US perspective on mycotoxin regulatory issues. Adv Exp Med Biol 504, 277-285.

Pirttilä, A. M., McIntyre, L. M., Payne, G. A. \& Woloshuk, C. P. (2004), Expression profile analysis of wild-type and $f c c 1$ mutant strains of Fusarium verticillioides during fumonisin biosynthesis. Fungal Genet Biol 41, 647-656.

Proctor, R. H., Desjardins, A. E., Plattner, R. D. \& Hohn, T. M. (1999), A polyketide synthase gene required for biosynthesis of fumonisin mycotoxins in Gibberella fujikuroi mating population A. Fungal Genet Biol 27, 100-112.

Proctor, R. H., Brown, D. W., Plattner, R. D. \& Desjardins, A. E. (2003). Co-expression of 15 contiguous genes delineates a fumonisin biosynthetic gene cluster in Gibberella moniliformis. Fungal Genet Biol 38, 237-249.

Saez, A., Apostolova, N., Gonzalez-Guzman, M., Gonzalez-Garcia, M. P., Nicolas, C., Lorenzo, O. \& Rodriguez, P. L. (2004). Gain-of function and loss-of-function phenotypes of the protein phosphatase $2 \mathrm{C}$ HAB1 reveal its role as a negative regulator of abscisic acid signaling. Plant J 37, 354-369.

Sagaram, U. S. \& Shim, W. B. (2007). Fusarium verticillioides GBB1, a gene encoding heterotrimeric $\mathrm{G}$ protein $\beta$ subunit, is associated with fumonisin $\mathrm{B}_{1}$ biosynthesis and hyphal development but not with fungal virulence. Mol Plant Pathol 8, 375-384.

Sagaram, U. S., Butchko, R. A. E. \& Shim, W. B. (2006). The putative monomeric G-protein $G B P 1$ is negatively associated with fumonisin $\mathrm{B}_{1}$ production in Fusarium verticillioides. Mol Plant Pathol 7, 381-389.

Sagot, I., Rodal, A. A., Moseley, J., Goode, B. L. \& Pellman, D. (2002) An actin nucleation mechanism mediated by Bnil and profiling. Nat Cell Biol 4, 626-631.

Sakumoto, N., Mukai, Y., Uchida, K., Kouchi, T., Kuwajima, J., Nakagawa, Y., Sugioka, S., Yamamoto, E., Furuyama, T. \& other authors (1999). A series of protein phosphatase gene disruptants in Saccharomyces cerevisiae. Yeast 15, 1669-1679.

Sambrook, J. \& Russell, D. W. (2001). Molecular Cloning: a Laboratory Manual. Cold Spring Harbor, NY: Cold Spring Harbor Laboratory.

Seo, J.-A., Proctor, R. H. \& Plattner, R. D. (2001). Characterization of four clustered and coregulated genes associated with fumonisin biosynthesis in Fusarium verticillioides. Fungal Genet Biol 34, 155-165.

Shaw, B. D. \& Upadhyay, S. (2005). Aspergillus nidulans swoK encodes an RNA binding protein that is important for cell polarity. Fungal Genet Biol 42, 862-872.

Shenolikar, S. (1994). Protein serine/threonine phosphatases-new avenues for cell regulation. Annu Rev Cell Biol 10, 55-86.

Sheu, Y.-J., Santos, B., Fortin, N., Costigan, C. \& Snyder, M. (1998). Spa2p interacts with cell polarity proteins and signaling components involved in yeast cell morphogenesis. Mol Cell Biol 18, 4053-4069.

Shim, W. B. \& Woloshuk, C. P. (1999). Nitrogen repression of fumonisin $\mathrm{B}_{1}$ biosynthesis in Gibberella fujikuroi. FEMS Microbiol Lett 177, 109-116

Shim, W. B. \& Woloshuk, C. P. (2001). Regulation of fumonisin B biosynthesis and conidiation in Fusarium verticillioides by a cyclin-like (C-type) gene, FCC1. Appl Environ Microbiol 67, 1607-1612.

Shimanuki, M., Kinoshita, N., Ohkura, H., Yoshida, T., Toda, T. \& Yanagida, M. (1993). Isolation and characterization of the fission yeast protein phosphatase gene ppe1+ involved in cell shape control and mitosis. Mol Biol Cell 4, 303-313.

Stark, M. J. R. (1996). Yeast protein serine/threonine phosphatase: multiple roles and diverse regulation. Yeast 12, 1647-1675.

Virag, A. \& Harris, S. D. (2006). Functional characterization of Aspergillus nidulans homologues of Saccharomyces cerevisiae Sap2 and Bud6. Eukaryot Cell 5, 881-895.

Wendland, J. (2003). Analysis of the landmark protein Bud3 of Ashbya gossypii reveals a novel role in septum construction. EMBO Rep 4, 200-204.

Yatzkan, E. \& Yarden, O. (1999). The B regulatory subunit of protein phosphatase $2 \mathrm{~A}$ is required for completion of macroconidiation and other developmental processes in Neurospora crassa. Mol Microbiol 31, 197-209.

Yatzkan, E., Szöor, B., Fehér, Z., Dombrádi, V. \& Yarden, O. (1998). Protein phosphatase 2A is involved in hyphal growth of Neurospora crassa. Mol Gen Genet 259, 523-531.

Yu, J.-H., Hamari, Z., Han, K.-H., Seo, J.-A., Reyes-Domínguez, Y. \& Scazzocchio, C. (2004). Double-joint PCR: a PCR-based molecular tool for gene manipulations in filamentous fungi. Fungal Genet Biol 41, 973-981.

Zabrocki, P., Van Hoof, C., Goris, J., Thevelein, J. M., Winderickx, J. \& Wera, S. (2002). Protein phosphatase $2 \mathrm{~A}$ on track for nutrientinduced signaling in yeast. Mol Microbiol 43, 835-842.

Zeke, T., Kókai, E., Szöor, B., Yatzkan, E., Yarden, O., Szirák, K., Fehér, Z., Bagossi, P., Gergely, P. \& Dombrádi, V. (2003). Expression of protein phosphatase 1 during the asexual development of Neurospora crassa. Comp Biochem Physiol B Biochem Mol Biol 134, $161-170$.

Edited by: J.-R. Xu 